\title{
La eliminación futura de la tuberculosis
}

\author{
The future elimination of tuberculosis
}

Los avances en el control global de la tuberculosis en los últimos 15 años han sido destacados. Se estima que entre el 2000 y el 2014 se han salvado cuarenta y tres millones de personas utilizando diagnósticos y tratamientos efectivos para esta enfermedad. Para el 201 I los objetivos de desarrollo del milenio planteados para el año 2015 se alcanzaron al detener y reversar la tendencia creciente de la incidencia de tuberculosis y probablemente se alcance el objetivo de disminuir en un 50\% las muertes con respecto a 1990, puesto que para el 201 I esta cifra estaba en el $41 \%$ [I]. A pesar de lo alentador de estas cifras, en el 2014 se enfermaron de tuberculosis 9.600 .000 personas y sigue siendo la enfermedad infecciosa con mayor mortalidad en el mundo en conjunto con el síndrome de inmunodeficiencia adquirida (SIDA), con un total de 1.500 .000 de muertos para el 2014 [I]. Es necesario tener en cuenta que la tuberculosis es una enfermedad asociada a la pobreza y a la inequidad, puesto que más del $90 \%$ de la carga de la enfermedad está en los países y las poblaciones más pobres. De hecho, los países más desarrollados han disminuido dramáticamente su carga de tuberculosis solo con mejorar el nivel de vida de su población y todo lo que esto conlleva, junto con el fortalecimiento de las actividades de diagnóstico y tratamiento.

Desde el 2016 la Organización Mundial de la Salud (OMS) plantea el objetivo de eliminar la tuberculosis, lo cual significa para el 2030, respecto a los niveles del 20 I5, disminuir la mortalidad en un $90 \%$ y el número de casos en un $80 \%$ y eliminar los costos catastróficos familiares que conlleva un paciente con tuberculosis [2]. Para alcanzar estos objetivos indudablemente se necesitará solucionar las deficiencias y limitaciones actuales que se tienen para diagnosticar pacientes y tratarlos; además, corregir las carencias en la financiación, no sólo de los programas de control sino también de la investigación necesaria para desarrollar nuevas alternativas para la prevención, el diagnóstico y el tratamiento.

En la última década los desarrollos en el diagnóstico y el tratamiento han tenido cambios como nunca antes en la historia del control de la enfermedad. En métodos diagnósticos es donde se observa un cambio radical en los paradigmas; la detección microscópica del bacilo, desarrollada hace más de 100 años, está siendo reemplazada en muchos países por nuevas tecnologías más sensibles, aunque no menos costosas, como es el caso del Xpert MTB/Rif ${ }^{\circledast}$ (Cepheid, California, Estados Unidos). Desde el 201 I esta técnica ha sido recomendada por la OMS como la primera opción de diagnóstico en varias condiciones clínicas. A pesar de su amplio uso actual, todavía tiene limitaciones en relación a las instalaciones que se necesitan y, particularmente, en cuanto al costo, el cual está todavía lejos de las posibilidades de muchos programas de control de la enfermedad.

Un desarrollo largamente esperado y que apenas hasta ahora se comienza a vislumbrar como posible, es el desarrollo de métodos que permitan hacer un diagnóstico de tuberculosis con el 
paciente presente, salvando una de las barreras más importantes de acceso al diagnóstico. Estos métodos, conocidos como POC (del inglés, point of care), permiten detectar biomarcadores propios de la enfermedad en líquidos corporales al lado del paciente. Este tipo de pruebas, como lo presenta el artículo de Jaramillo-Grajales y colaboradores en este número de Medicina \& Laboratorio, basadas en tecnologías como los sensores piezoeléctricos, tienen la potencialidad de cumplir las premisas de rapidez y bajo costo de una nueva prueba diagnóstica de tuberculosis, necesarias para cumplir con los objetivos del plan Fin de la Tuberculosis de la OMS [2].

El reto entonces actual radica en que los nuevos medicamentos y las tecnologías diagnosticas que se desarrollen estén al alcance de los pacientes que realmente las necesitan y sean utilizadas eficientemente. Solo así, lo que ofrece el desarrollo científico como soluciones de salud y, en este caso, para el diagnóstico de la tuberculosis, puede tener el mayor impacto para eliminar la enfermedad en el futuro próximo.

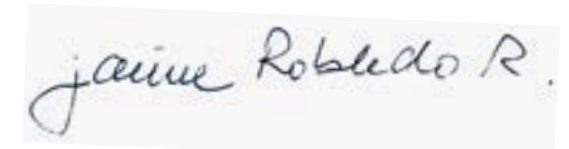

\section{Jaime Robledo Restrepo. MD, PhD.}

Corporación para Investigaciones Biológicas

Universidad Pontificia Bolivariana

Medellín, Colombia, agosto 2015

\section{Bibliografía}

I. World Health Organization. Global tuberculosis report 2015 (ed 20a). Francia: World Health Organization; 2015.
2. Uplekar M, Weil D, Lonnroth K, Jaramillo E, Lienhardt C, Dias HM, et al. WHO's new End TB Strategy. Lancet 2015; 385: |799-180|. 